01

\title{
Приближенные формулы, описывающие профили лежащих и висящих капель в случаях малых чисел Бонда и сильной смачиваемости
}

\author{
(C) Е.В. Галактионов, ${ }^{1}$ Н.Е. Галактионова, ${ }^{2}$ Э.А. Тропп ${ }^{1}$ \\ ${ }^{1}$ Физико-технический институт им. А.Ф. Иоффре, \\ 194021 Санкт-Петербург, Россия \\ ${ }^{2}$ Санкт-Петербургский политехнический университет Петра Великого, \\ 195251 Санкт-Петербург, Россия \\ e-mail: evgalakt@mail.ru
}

(Поступило в Редакцию 25 мая 2016 г.)

Даны вариационные постановки задач о лежащей и висящей каплях с учетом силы тяжести в осесимметричном случае. Получены приближенные формулы, описывающие профили поверхностей этих капель при малых числах Бонда - асимптотическим методом, а при условии сильной смачиваемости - методом линеаризации.

\section{Введение}

Результаты теоретического исследования профиля поверхности жидкой капли малого объема на твердой поверхности при наличии зоны трехфазного контакта представляют ценную информацию для решения многих научных и технологических проблем [1]. Задачи о равновесии капиллярных поверхностей являются базой для изучения формы жидких менисков в процессе роста кристаллов по способу Степанова [2].

Как известно, использование вариационной постановки задачи позволяет получить и дифференциальные уравнения, и граничные условия (естественные условия экстремума функционала). В своей монографии [3] H.M. Гюнтер пишет: „.. .вариационная постановка задачи способствует обнаружению законов, управляющих явлением.“"

В работе [4] сформулирована вариационная постановка основных задач этого класса: капля на твердой поверхности, капля на капле другой жидкости, капля с частично ограненной поверхностью. В работах $[1,5,6]$ проведено исследование задачи о капле, лежащей на твердой поверхности с учетом действия силы тяжести, кроме того, в [1] рассмотрена и задача о висящей капле. В [5] с помощью метода линеаризации получены приближенные формулы, описывающие профиль лежащей капли при условии сильной смачиваемости в декартовых координатах (для бесконечной балки с сечением в виде профиля капли).

В настоящей работе рассматрены осесимметричные задачи о лежащей и висящей каплях в цилиндрических координатах с учетом силы тяжести, даны их вариационные постановки. Построены приближенные решения этих задач для случая малых чисел Бонда (найдено нулевое и первое приближения асимптотического разложения). При условии сильной смачиваемости методом линеаризации получены приближенные формулы, справедливые для любого значения числа Бонда. Построена асимптотика решений при угле смачивания, стремящемся к нулю.

Исследование таких задач актуально для изучения процессов, имеющих место при затравлении кристаллов, выращиваемых из расплава [2].

\section{Лежащая капля. Постановка задачи}

Рассмотрим случай капли лежащей на твердой поверхности при наличии трех фаз и с учетом силы тяжести (рис. 1). Ввиду осевой симметрии будем решать задачу о нахождении профиля капли в цилиндрической системе координат. Область $\Omega$, занимаемая каплей на твердой поверхности, представляет собой круг радиуса $r_{1}\left(r_{1}-\right.$ искомая величина). Объем капли - заданная величина $V_{1}$ и, следовательно,

$$
I_{1}\left\{u_{1}(r)\right\}=2 \pi \int_{0}^{r_{1}} u_{1}(r) r d r=V_{1},
$$

где $u_{1}(r)$ - искомая функция, описывающая профиль поверхности лежащей капли. Будем рассматривать только случай однозначной функции $u_{1}(r)$.

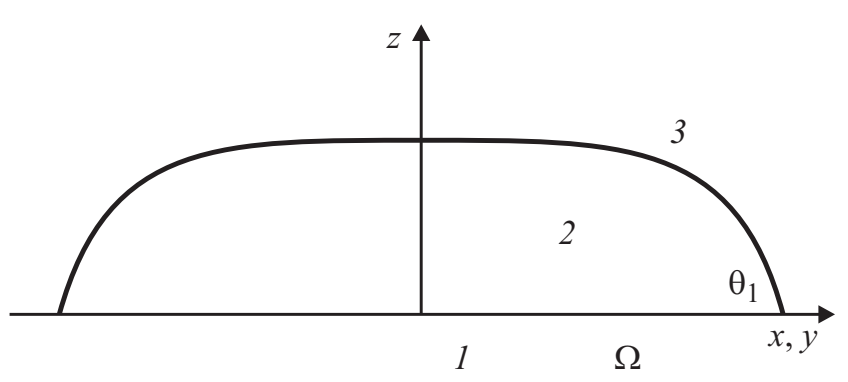

Рис. 1. Капля, лежащая на твердой поверхности. 1 - твердая среда, 2 - жидкая, 3 - газообразная; $\theta_{1}-$ угол смачивания. 
Введем в рассмотрение функционал, включающий в себя поверхностную энергию и энергию силы тяжести. Поверхностная энергия в свою очередь состоит из части, соответствующей свободной поверхности капли, и части, соответствующей контакту жидкости с твердым телом. Таким образом, следуя [5], функционал можно записать в виде

$$
\begin{aligned}
J_{1}\left\{u_{1}(r)\right\}= & 2 \pi \int_{0}^{r_{1}}\left\{\alpha_{23} \sqrt{1+\left(u_{1}^{\prime}\right)^{2}}\right. \\
& \left.+\alpha_{12}-\alpha_{13}+\frac{1}{2} g \rho u_{1}^{2}\right\} r d r
\end{aligned}
$$

где $\alpha_{i j}-$ поверхностные натяжения между различными средами: твердой (1), жидкой (2) и газообразной (3), $g$ - ускорение силы тяжести, $\rho$ - плотность жидкости.

Итак, надо найти экстремум функционала (2) при условии, что функционал (1) принимает заданное значение, т. е. приходим к изопериметрической задаче [3] со свободной границей $\left(r_{1}-\right.$ искомая величина). Для ее решения необходимо минимизировать функционал

$$
J_{1}+\lambda_{1} I_{1}=\int_{0}^{r_{1}} H_{1}\left(r, u_{1}(r), u_{1}^{\prime}(r)\right) d r,
$$

где

$$
\begin{aligned}
H_{1}\left(r, u_{1}(r), u_{1}^{\prime}(r)\right)= & 2 \pi\left\{\alpha_{23} \sqrt{1+\left(u_{1}^{\prime}\right)^{2}}+\alpha_{12}-\alpha_{13}\right. \\
& \left.+\frac{1}{2} g \rho u_{1}^{2}+\lambda_{1} u_{1}\right\} r .
\end{aligned}
$$

Уравнение Эйлера для функционала (3) будет иметь вид

$$
\frac{d}{d r}\left(\frac{\alpha_{23} r u_{1}^{\prime}}{\sqrt{1+\left(u_{1}^{\prime}\right)^{2}}}\right)=g \rho r u_{1}+\lambda_{1} r .
$$

Кроме условия постоянства объема (1) при этом должны еще выполняться условия

$$
u_{1}\left(r_{1}\right)=0, \quad u_{1}^{\prime}(0)=0
$$

и условие трансверсальности [3]

$$
\left.\left\{H_{1}-u_{1}^{\prime} \frac{\partial H_{1}}{\partial u_{1}^{\prime}}\right\}\right|_{r=r_{1}}=0,
$$

которое с учетом первого из условий (5) дает известное соотношение, связывающее угол смачивания и поверхностные натяжения в точке контакта трех фаз

$$
\left.\cos \theta_{1} \equiv \frac{1}{\sqrt{1+\left(u_{1}^{\prime}\right)^{2}}}\right|_{r=r_{1}}=\frac{\alpha_{13}-\alpha_{12}}{\alpha_{23}} \equiv \alpha_{1} .
$$

Наличие условия трансверсальности связано с тем, что решается вариационная задача с подвижной границей (точка с координатами $\left(r_{1}, 0\right)$ может двигаться по оси абсцисс). Ввиду предположения об однозначности функции, описывающей профиль капли, угол $\theta_{1}$ не должен превосходить $90^{\circ}$ и правая часть (6) положительна.

Приведем задачу к безразмерному виду с помощью введения следующих масштабов:

$$
\xi=\frac{r}{V_{1}^{1 / 3}}, \quad w_{1}=\frac{u_{1}}{V_{1}^{1 / 3}}, \quad \tilde{\lambda}_{1}=\frac{\lambda_{1} V_{1}^{1 / 3}}{\alpha_{23}}
$$

$\left(V_{1}^{1 / 3}\right.$ - характерный размер капли) и введем обозначение

$$
B_{1}=\frac{g \rho V_{1}^{2 / 3}}{\alpha_{23}} \text {. }
$$

Безразмерная постоянная $B_{1}$ - число Бонда (см. [1]).

В новых переменных задача будет иметь следующий вид:

уравнение Эйлера

$$
\frac{d}{d \xi}\left(\frac{\xi w_{1}^{\prime}(\xi)}{\sqrt{1+\left(w_{1}^{\prime}(\xi)\right)^{2}}}\right)=B_{1} \xi w_{1}(\xi)+\tilde{\lambda}_{1} \xi,
$$

условия

$$
\begin{gathered}
\int_{0}^{\xi_{1}} w_{1}(\xi) \xi d \xi=\frac{1}{2 \pi}, \\
w_{1}\left(\xi_{1}\right)=0, \quad w_{1}^{\prime}(0)=0, \\
\left.\frac{1}{\sqrt{1+\left(w_{1}^{\prime}\right)^{2}}}\right|_{\xi=\xi_{1}}=\alpha_{1},
\end{gathered}
$$

где $\xi_{1}-$ значение новой независимой переменной, соответствующее значению $r_{1}$.

\section{Лежащая капля. Асимптотический метод}

Построим решение задачи о лежащей капле для случая малых чисел Бонда. Малые числа Бонда соответствуют жидким каплям малого объема, например, для случая капли расплава $\mathrm{Al}_{2} \mathrm{O}_{3}$ [2] число Бонда меньше 0.1, если диаметр капли меньше двух миллиметров.

Итак, пусть $B_{1}$ - малый параметр задачи (7)-(10). Будем искать решение в виде ряда по степеням малого параметра $B_{1}$ :

$$
w_{1}(\xi)=w_{10}(\xi)+w_{11}(\xi) B_{1}+w_{12}(\xi) B_{1}^{2}+\ldots
$$

Искомые величины $\xi_{1}, \tilde{\lambda}_{1}$ также будем искать в виде разложений по степеням $B_{1}$ :

$$
\begin{aligned}
& \xi_{1}=\xi_{10}+\xi_{11} B_{1}+\xi_{12} B_{1}^{2}+\ldots \\
& \tilde{\lambda}_{1}=\tilde{\lambda}_{10}+\tilde{\lambda}_{11} B_{1}+\tilde{\lambda}_{12} B_{1}^{2}+\ldots
\end{aligned}
$$


Подставив эти разложения в уравнение (7) и приравняв коэффициенты при одинаковых степенях малого параметра $B_{1}$ в левых и правых частях, получим

$$
\begin{gathered}
\frac{d}{d \xi}\left(\frac{\xi w_{10}^{\prime}(\xi)}{\sqrt{1+\left(w_{10}^{\prime}(\xi)\right)^{2}}}\right)=\tilde{\lambda}_{10} \xi, \\
\frac{d}{d \xi}\left(\frac{\xi w_{11}^{\prime}(\xi)}{\left[1+\left(w_{10}^{\prime}(\xi)\right)^{2}\right]^{3 / 2}}\right)=w_{10}(\xi) \xi+\tilde{\lambda}_{11} \xi
\end{gathered}
$$

- уравнения нулевого и первого приближений соответственно. Условие постоянства объема капли (условие (8)) даст в этих приближениях следующие условия:

$$
\begin{gathered}
\int_{0}^{\xi_{10}} w_{10}(\xi) \xi d \xi=\frac{1}{2 \pi}, \\
\int_{0}^{\xi_{10}} w_{11}(\xi) \xi d \xi+w_{10}\left(\xi_{10}\right) \xi_{10} \xi_{11}=0 .
\end{gathered}
$$

Первое из условий (9) даст в нулевом и первом приближениях соответственно

$$
\begin{gathered}
w_{10}\left(\xi_{10}\right)=0, \\
w_{11}\left(\xi_{10}\right)+w_{10}^{\prime}\left(\xi_{10}\right) \xi_{11}=0 .
\end{gathered}
$$

Второе из условий (9) даст в нулевом и первом приближениях соответственно

$$
\begin{aligned}
& w_{10}^{\prime}(0)=0 \\
& w_{11}^{\prime}(0)=0 .
\end{aligned}
$$

И наконец, четвертое условие (условие (10)) даст, соответственно

$$
\begin{gathered}
\frac{1}{\sqrt{1+\left(w_{10}^{\prime}\left(\xi_{10}\right)\right)^{2}}}=\alpha_{1}, \\
w_{11}^{\prime}\left(\xi_{10}\right)+w_{10}^{\prime \prime}\left(\xi_{10}\right) \xi_{11}=0 .
\end{gathered}
$$

Таким образом, получили задачу нулевого приближения (11), (13), (15), (17), (19) и задачу первого приближения (12), (14), (16), (18), (20).

Решив задачу нулевого приближения, получим

$$
\begin{gathered}
\tilde{\lambda}_{10}=-2\left\{\frac{\pi\left(1-\alpha_{1}\right)^{2}\left(2+\alpha_{1}\right)}{3}\right\}^{1 / 3}, \\
\xi_{10}=-2\left(\tilde{\lambda}_{10}\right)^{-1} \sqrt{1-\alpha_{1}^{2}},
\end{gathered}
$$

а профиль лежащей капли в нулевом приближении будет определяться выражением

$$
w_{10}(\xi)=2\left(\tilde{\lambda}_{10}\right)^{-1}\left\{\alpha_{1}-\sqrt{1-\left(0.5 \tilde{\lambda}_{10} \xi\right)^{2}}\right\} .
$$

Перейдем к решению задачи первого приближения. В результате получим систему двух линейных алгебраических уравнений для определения $\tilde{\lambda}_{11}$ и $\xi_{11}$ :

$$
\begin{gathered}
\tilde{\lambda}_{11}=\frac{1}{\xi_{10}^{2}}\left[2 \sqrt{1-\alpha_{1}^{2}} \xi_{11}-\frac{1}{\pi}\right], \\
\xi_{11}=-\frac{1}{4 \pi\left(1-\alpha_{1}^{2}\right)^{3 / 2}\left(2+\alpha_{1}\right)}\left[3 \tilde{\lambda}_{10} \tilde{\lambda}_{11}+4 \alpha_{1}-4\right] .
\end{gathered}
$$

Решив эту систему, найдем $\tilde{\lambda}_{11}$ и $\xi_{11}$ :

$$
\begin{gathered}
\tilde{\lambda}_{11}=2 \alpha_{1}\left(3+\alpha_{1}\right)\left(\alpha_{1}-1\right)\left[3 \tilde{\lambda}_{10}\right. \\
\left.+2 \pi\left(1-\alpha_{1}^{2}\right)\left(2+\alpha_{1}\right) \xi_{10}^{2}\right]^{-1}, \\
\xi_{11}=\left[3 \tilde{\lambda}_{10}+4 \pi\left(1-\alpha_{1}\right) \xi_{10}^{2}\right] \\
\left\{2 \pi \sqrt{1-\alpha_{1}^{2}}\left[3 \tilde{\lambda}_{10}+2 \pi\left(1-\alpha_{1}^{2}\right)\left(2+\alpha_{1}\right) \xi_{10}^{2}\right]\right\}^{-1},
\end{gathered}
$$

a первая поправка для профиля лежащей капли будет иметь вид

$$
\begin{aligned}
w_{11}(\xi)= & \frac{\sqrt{1-\alpha_{1}^{2}}}{\alpha_{1}} \xi_{11}+\frac{2 \tilde{\lambda}_{10}^{-3}}{3}\left\{\left(\sqrt{1-\left(0.5 \tilde{\lambda}_{10} \xi\right)^{2}}-\alpha_{1}\right)\right. \\
& \times\left(\frac{4}{\alpha_{1} \sqrt{1-\left(0.5 \tilde{\lambda}_{10} \xi\right)^{2}}}-3\left(\tilde{\lambda}_{10} \tilde{\lambda}_{11}+2 \alpha_{1}\right)\right) \\
& \left.+4 \ln \left(\frac{1+\sqrt{1-\left(0.5 \tilde{\lambda}_{10} \xi\right)^{2}}}{1+\alpha_{1}}\right)\right\} .
\end{aligned}
$$

Тогда приближенная формула, описывающая форму поверхности лежащей капли с точностью до членов второго порядка малости относительно числа Бонда $B_{1}$, может быть записана в следующей форме:

$$
w_{1}(\xi)=w_{10}(\xi)+w_{11}(\xi) B_{1} .
$$

\section{Лежащая капля. Метод линеаризации}

Построим решение задачи о лежащей капле (задачу (7)-(10)) для случая сильной смачиваемости методом линеаризации. Условие (10) перепишем в виде

$$
w_{1}^{\prime}\left(\xi_{1}\right)=-\frac{\sqrt{1-\alpha_{1}^{2}}}{\alpha_{1}} \equiv-\gamma_{1} .
$$

Другими словами, рассмотрим случай

$$
0 \leq \gamma_{1} \ll 1
$$

Тогда уравнение (7) можно заменить на линеаризованное уравнение

$$
\frac{d}{d \xi}\left(\xi w_{1}^{\prime}(\xi)\right)=B_{1} \xi w_{1}(\xi)+\tilde{\lambda}_{1} \xi
$$


Общее решение этого уравнения имеет вид

$$
w_{1}(\xi)=C_{1} I_{0}\left(\sqrt{B_{1}} \xi\right)+C_{2} K_{0}\left(\sqrt{B_{1}} \xi\right)-\frac{\tilde{\lambda}_{1}}{B_{1}},
$$

где $C_{1}$ и $C_{2}$ - произвольные постоянные. С учетом второго из условий (9) получим $C_{2}=0$ и, следовательно,

$$
w_{1}(\xi)=C_{1} I_{0}\left(\sqrt{B_{1}} \xi\right)-\frac{\tilde{\lambda}_{1}}{B_{1}}, \quad w_{1}^{\prime}(\xi)=C_{1} \sqrt{B_{1}} I_{1}\left(\sqrt{B_{1}} \xi\right)
$$

Отсюда с учетом условия (27) и первого из условий (9) находим

$$
\begin{gathered}
C_{1}=-\frac{\gamma_{1}}{\sqrt{B_{1}} I_{1}\left(\sqrt{B_{1}} \xi_{1}\right)}, \\
C_{1} I_{0}\left(\sqrt{B_{1}} \xi_{1}\right)=\frac{\tilde{\lambda}_{1}}{B_{1}} .
\end{gathered}
$$

Из (33) с учетом (32) получим выражение для нахождения множителя Лагранжа $\tilde{\lambda}_{1}$, а именно

$$
\tilde{\lambda}_{1}=-\gamma_{1} \sqrt{B_{1}} \frac{I_{0}\left(\sqrt{B_{1}} \xi_{1}\right)}{I_{1}\left(\sqrt{B_{1}} \xi_{1}\right)} .
$$

Осталось найти $\xi_{1}$. Подставим в (8) выражение для $w_{1}(\xi)$ из (31) и, выполнив интегрирование, получим трансцендентное уравнение для нахождения величины $\xi_{1}$ :

$$
\left\{\frac{I_{0}\left(\sqrt{B_{1}} \xi_{1}\right)}{I_{1}\left(\sqrt{B_{1}} \xi_{1}\right)} \sqrt{B_{1}} \xi_{1}-2\right\} \xi_{1}=\frac{B_{1}}{\pi \gamma_{1}} .
$$

Решив это уравнение, найдем $\xi_{1}$, далее по формуле (34) определим $\tilde{\lambda}_{1}$, а по формуле $(32)-C_{1}$. Приближенную формулу для профиля лежащей капли, полученную в результате первого шага процесса линеаризации, даст (31).

Далее, подставим полученное нулевое приближение для функции $w_{1}(\xi)$ в знаменатель левой части уравнения (7). Получим линейное обыкновенное дифференциальное уравнение второго порядка для нахождения следующего приближения процесса линеаризации и повторим весь процесс, описанный выше. Таков алгоритм процесса линеаризации для случая капли, лежащей на твердой поверхности.

Рассмотрим случай $\gamma_{1}=0$ (нулевой угол смачивания). В этом случае для $\xi_{1}<+\infty$ из (32) следует $C_{1}=0$, из (33) $-\tilde{\lambda}_{1}=0$ и (31) дает $w_{1}(\xi) \equiv 0$, но условие $(8)$ при этом не выполняется и $w_{1}(\xi) \equiv 0$ не есть решение исходной задачи.

Построим асимптотику решения задачи первого шага процесса линеаризации при $\gamma_{1} \rightarrow 0$. Из трансцендентного уравнения (35) видно, что при $\gamma_{1} \rightarrow+0$ необходимо $\xi_{1} \rightarrow+\infty$, а из асимптотики модифицированных функций Бесселя при больших значениях аргумента следует, что

$$
\left.\frac{I_{0}\left(\sqrt{B_{1}} \xi_{1}\right)}{I_{1}\left(\sqrt{B_{1}} \xi_{1}\right)}\right|_{\xi_{1} \rightarrow+\infty} \rightarrow+1
$$

Главным членом в левой части уравнения (35) при этом будет член $\sqrt{B_{1}} \xi_{1}^{2}$, следовательно, $\xi_{1} \sim D_{1} / \sqrt{\gamma_{1}}$, где постоянная $D_{1}=\left(B_{1}\right)^{1 / 4} / \sqrt{\pi}$. Далее, после того как найдена асимптотика значения $\xi_{1}$ при $\gamma_{1} \rightarrow+0$, выражения (32) и (34) дадут асимптотику величин $C_{1}, \tilde{\lambda}_{1}$, а именно

$C_{1} \sim-\frac{\sqrt{2}(\pi)^{1 / 4}}{\left(B_{1}\right)^{1 / 8} \exp \left(\left(B_{1}\right)^{3 / 4} / \sqrt{\pi \gamma_{1}}\right)}\left(\gamma_{1}\right)^{3 / 4}, \quad \tilde{\lambda}_{1} \sim-\sqrt{B_{1}} \gamma_{1}$ при $\gamma_{1} \rightarrow+0$.

Следовательно, асимптотика при $\gamma_{1} \rightarrow+0$ для профиля лежащей капли после первого шага процесса линеаризации будет иметь вид

$$
w_{1}(\xi) \sim-\frac{\sqrt{2}(\pi)^{1 / 4}\left(\gamma_{1}\right)^{3 / 4}}{\left(B_{1}\right)^{1 / 8} \exp \left(\left(B_{1}\right)^{3 / 4} / \sqrt{\pi \gamma_{1}}\right)} I_{0}\left(\sqrt{B_{1}} \xi\right)+\frac{\gamma_{1}}{\sqrt{B_{1}}} .
$$

Видно, что максимум модуля асимптотического представления решения в каждой точке линейно стремится к нулю при $\gamma_{1} \rightarrow+0$.

\section{Висящая капля. Постановка задачи}

Перейдем к рассмотрению висящей капли (см. рис. 2). Пусть капля висит на твердой поверхности (плоскость $z=h, h>0)$. Поверхностные натяжения между твердой и жидкой, твердой и газообразной средами на этой плоскости $\alpha_{42}$ и $\alpha_{43}$ соответственно. Область контакта висящей капли и плоскости $z=h$ (область $\Omega$ ) представляет собой круг радиуса $r_{2}\left(r_{2}-\right.$ искомая величина). Объем висящей капли - заданная величина $V_{2}$ и, следовательно,

$$
I_{2}\left\{u_{2}(r)\right\}=2 \pi \int_{0}^{r_{2}}\left(h-u_{2}(r)\right) r d r=V_{2},
$$

где $u_{2}(r)$ - искомая функция, описывающая профиль поверхности висящей капли.

Далее, по аналогии с рассмотренным выше случаем лежащей капли введем в рассмотрение функционал, включающий в себя поверхностную энергию и энергию силы тяжести. Поверхностная энергия в свою очередь будет состоять из части, соответствующей свободной поверхности капли, и части, соответствующей контакту

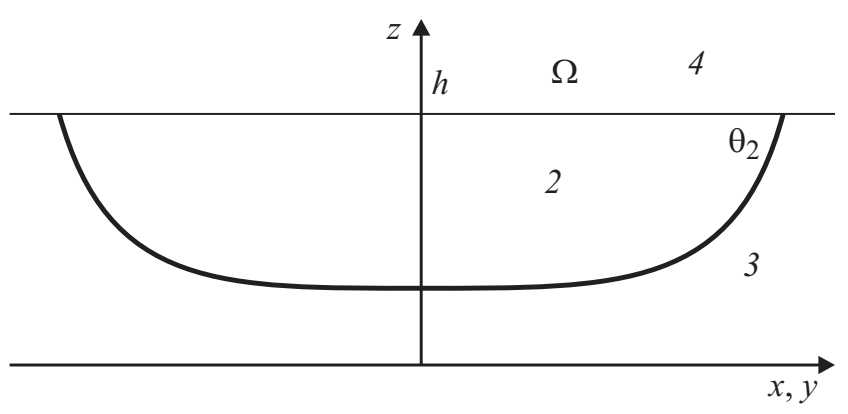

Рис. 2. Капля, висящая на твердой поверхности. 4 - твердая среда, 2 - жидкая, 3 - газообразная; $\theta_{2}-$ угол смачивания. 
жидкости с твердым телом. Этот случай будет отличаться от рассмотренного выше только тем, что в уравнение энергетического баланса энергия силы тяжести войдет с противоположным знаком. Таким образом, функционал будет иметь следующий вид:

$$
\begin{aligned}
J_{2}\left\{u_{2}(r)\right\}= & 2 \pi \int_{0}^{r_{2}}\left\{\alpha_{23} \sqrt{1+\left(u_{2}^{\prime}\right)^{2}}+\alpha_{42}-\alpha_{43}\right. \\
& \left.-\frac{1}{2} g \rho\left(h-u_{2}\right)^{2}\right\} r d r .
\end{aligned}
$$

Надо найти экстремум функционала (37) при условии, что функционал (36) принимает заданное значение, т.е. приходим к изопериметрической задаче со свободной границей $\left(r_{2}-\right.$ искомая величина). Для ее решения необходимо минимизировать функционал

$$
J_{2}+\lambda_{2} I_{2}=\int_{0}^{r_{2}} H_{2}\left(r, u_{2}(r), u_{2}^{\prime}(r)\right) d r
$$

где

$$
\begin{aligned}
H_{2}\left(r, u_{2}(r), u_{2}^{\prime}(r)\right)= & 2 \pi\left\{\alpha_{23} \sqrt{1+\left(u_{2}^{\prime}\right)^{2}}+\alpha_{42}-\alpha_{43}\right. \\
& \left.-\frac{1}{2} g \rho\left(h-u_{2}\right)^{2}+\lambda_{2}\left(h-u_{2}\right)\right\} r .
\end{aligned}
$$

Уравнение Эйлера для функционала (38) будет иметь вид

$$
\frac{d}{d r}\left(\frac{\alpha_{23} r u_{2}^{\prime}}{\sqrt{1+\left(u_{2}^{\prime}\right)^{2}}}\right)=g \rho\left(h-u_{2}\right) r-\lambda_{2} r .
$$

Кроме условия (36) должны выполняться еще три: условия

$$
u_{2}\left(r_{2}\right)=h, \quad u_{2}^{\prime}(0)=0
$$

и условие трансверсальности

$$
\left.\left\{H_{2}-u_{2}^{\prime} \frac{\partial H_{2}}{\partial u_{2}^{\prime}}\right\}\right|_{r=r_{2}}=0 .
$$

Это условие с учетом первого из условий (40) можно переписать в следующей форме:

$$
\left.\cos \theta_{2} \equiv \frac{1}{\sqrt{1+\left(u_{2}^{\prime}\right)^{2}}}\right|_{r=r_{2}}=\frac{\alpha_{43}-\alpha_{42}}{\alpha_{23}} \equiv \alpha_{2} .
$$

Имеем четыре условия, включая условие постоянства объема, и четыре неизвестные постоянные: $r_{2}, \lambda_{2}$ и две постоянные интегрирования уравнения Эйлера. В этой задаче кроме параметра $\alpha_{2}$ появился еще один параметр - высота $h$ (в безразмерном виде $\tilde{h}=h / V_{2}^{1 / 3}$ ).

Далее, аналогично случаю лежащей капли, приведем задачу к безразмерному виду и введем постоянную Бонда $B_{2}$.

В новых переменных задача будет иметь следующий вид: уравнение Эйлера

$$
\frac{d}{d \xi}\left(\frac{\xi w_{2}^{\prime}(\xi)}{\sqrt{1+\left(w_{2}^{\prime}(\xi)\right)^{2}}}\right)=B_{2}\left(\tilde{h}-w_{2}(\xi)\right) \xi-\tilde{\lambda}_{2} \xi,
$$

условия

$$
\begin{aligned}
& \int_{0}^{\xi_{2}}\left(\tilde{h}-w_{2}(\xi)\right) \xi d \xi=\frac{1}{2 \pi}, \\
& w_{2}\left(\xi_{2}\right)=\tilde{h}, \quad w_{2}^{\prime}(0)=0, \\
& w_{2}^{\prime}\left(\xi_{2}\right)=\frac{\sqrt{1-\alpha_{2}^{2}}}{\alpha_{2}} \equiv \gamma_{2},
\end{aligned}
$$

где $\xi_{2}-$ значение новой независимой переменной, соответствующее значению $r_{2}$.

\section{Висящая капля. Асимптотический метод}

Построим решение задачи о висящей капле для случая малых чисел Бонда. Итак, пусть $B_{2}$ - малый параметр задачи (43)-(46). Будем искать решение в виде ряда по степеням малого параметра $B_{2}$ аналогично случаю лежащей капли.

В результате получим

$$
\begin{gathered}
\frac{d}{d \xi}\left(\frac{\xi w_{20}^{\prime}(\xi)}{\sqrt{1+\left(w_{20}^{\prime}(\xi)\right)^{2}}}\right)=-\tilde{\lambda}_{20} \xi, \\
\frac{d}{d \xi}\left(\frac{\xi w_{21}^{\prime}(\xi)}{\left[1+\left(w_{20}^{\prime}(\xi)\right)^{2}\right]^{3 / 2}}\right)=\left(\tilde{h}-w_{20}(\xi)\right) \xi-\tilde{\lambda}_{21} \xi
\end{gathered}
$$

- уравнения нулевого и первого приближений соответственно.

Условие постоянства объема капли (условие (44)) даст в этих приближениях следующие условия:

$$
\begin{gathered}
\int_{0}^{\xi_{20}}\left(\tilde{h}-w_{20}(\xi)\right) \xi d \xi=\frac{1}{2 \pi}, \\
\int_{0}^{\xi_{20}} w_{21}(\xi) \xi d \xi=\left[\tilde{h}-w_{20}\left(\xi_{20}\right)\right] \xi_{20} \xi_{21} .
\end{gathered}
$$

Первое из условий (45) даст в нулевом и первом приближениях соответственно

$$
\begin{gathered}
w_{20}\left(\xi_{20}\right)=\tilde{h}, \\
w_{21}\left(\xi_{20}\right)+w_{20}^{\prime}\left(\xi_{20}\right) \xi_{21}=0 .
\end{gathered}
$$

Второе из условий (45) даст в нулевом и первом приближениях соответственно

$$
\begin{aligned}
& w_{20}^{\prime}(0)=0, \\
& w_{21}^{\prime}(0)=0 .
\end{aligned}
$$


И наконец, четвертое условие (условие (46)) даст соответственно

$$
\begin{gathered}
w_{20}^{\prime}\left(\xi_{20}\right)=\frac{\sqrt{1-\alpha_{2}^{2}}}{\alpha_{2}}, \\
w_{21}^{\prime}\left(\xi_{20}\right)+w_{20}^{\prime \prime}\left(\xi_{20}\right) \xi_{21}=0 .
\end{gathered}
$$

Таким образом, получили задачу нулевого приближения (47), (49), (51), (53), (55) и задачу первого приближения (48), (50), (52), (54), (56) для висящей капли.

Решив задачу нулевого приближения, получим

$$
\begin{gathered}
\tilde{\lambda}_{20}=-2\left\{\frac{\pi\left(1-\alpha_{2}\right)^{2}\left(2+\alpha_{2}\right)}{3}\right\}^{1 / 3}, \\
\xi_{20}=-2\left(\tilde{\lambda}_{20}\right)^{-1} \sqrt{1-\alpha_{2}^{2}},
\end{gathered}
$$

а профиль поверхности висящей капли будет описываться формулой

$$
w_{20}(\xi)=\tilde{h}-2\left(\tilde{\lambda}_{20}\right)^{-1}\left\{\alpha_{2}-\sqrt{1-\left(0.5 \tilde{\lambda}_{20} \xi\right)^{2}}\right\} .
$$

Нетрудно заметить, что если выполнено условие $\alpha_{2}=\alpha_{1}$, то при построении нулевых приближений для лежащей и висящей капель будем иметь $\tilde{\lambda}_{20}=\tilde{\lambda}_{10}$, $\xi_{20}=\xi_{10}$ и соответствующие профили будут симметричны относительно прямой $f(\xi)=\tilde{h} / 2$.

Приступим к решению задачи первого приближения для висящей капли. В результате получим систему двух линейных алгебраических уравнений для определения $\tilde{\lambda}_{21}$ и $\xi_{21}$ :

$$
\begin{gathered}
\tilde{\lambda}_{21}=\frac{1}{\xi_{20}^{2}}\left[2 \sqrt{1-\alpha_{2}^{2}} \xi_{21}+\frac{1}{\pi}\right], \\
\xi_{21}=-\frac{1}{4 \pi\left(1-\alpha_{2}^{2}\right)^{3 / 2}\left(2+\alpha_{2}\right)}\left[3 \tilde{\lambda}_{20} \tilde{\lambda}_{21}-4 \alpha_{2}+4\right] .
\end{gathered}
$$

Решив эту систему, найдем $\tilde{\lambda}_{21}$ и $\xi_{21}$ :

$$
\begin{gathered}
\tilde{\lambda}_{21}=-2 \alpha_{2}\left(3+\alpha_{2}\right)\left(\alpha_{2}-1\right)\left[3 \tilde{\lambda}_{20}\right. \\
\left.+2 \pi\left(1-\alpha_{2}^{2}\right)\left(2+\alpha_{2}\right) \xi_{20}^{2}\right]^{-1}, \\
\xi_{21}=-\left[3 \tilde{\lambda}_{20}+4 \pi\left(1-\alpha_{2}\right) \xi_{20}^{2}\right] \\
\left\{2 \pi \sqrt{1-\alpha_{2}^{2}}\left[3 \tilde{\lambda}_{20}+2 \pi\left(1-\alpha_{2}^{2}\right)\left(2+\alpha_{2}\right) \xi_{20}^{2}\right]\right\}^{-1},
\end{gathered}
$$

а первая поправка для профиля висящей капли будет иметь вид

$$
\begin{aligned}
w_{21}(\xi)= & -\frac{\sqrt{1-\alpha_{2}^{2}}}{\alpha_{2}} \xi_{21}+\frac{2 \tilde{\lambda}_{20}^{-3}}{3}\left\{\left(\sqrt{1-\left(0.5 \tilde{\lambda}_{20} \xi\right)^{2}}-\alpha_{2}\right)\right. \\
& \times\left[\frac{4}{\alpha_{2} \sqrt{1-\left(0.5 \tilde{\lambda}_{20} \xi\right)^{2}}}+3\left(\tilde{\lambda}_{20} \tilde{\lambda}_{21}-2 \alpha_{2}\right)\right] \\
& \left.+4 \ln \left(\frac{1+\sqrt{1-\left(0.5 \tilde{\lambda}_{20} \xi\right)^{2}}}{1+\alpha_{2}}\right)\right\} .
\end{aligned}
$$

Видно, что в случае $\alpha_{2}=\alpha_{1}$ будем иметь $\tilde{\lambda}_{21}=-\tilde{\lambda}_{11}$, $\xi_{21}=-\xi_{11}$ и первые поправки к профилям лежащей и висящей капель будут совпадать.

Приближенная формула, описывающая форму поверхности висящей капли с точностью до членов второго порядка малости относительно числа Бонда $B_{2}$, может быть записана в следующей форме:

$$
w_{2}(\xi)=w_{20}(\xi)+w_{21}(\xi) B_{2} .
$$

\section{Висящая капля. Метод линеаризации}

Построим решение задачи о висящей капле (задачи (43)-(46)) для случая сильной смачиваемости методом линеаризации. Заметим, что при использовании такого подхода нет ограничений на величину числа Бонда (аналогичная ситуация имеет место и для лежащей капли).

Итак, рассмотрим случай

$$
0 \leq \gamma_{2} \ll 1 .
$$

Тогда уравнение (43) можно заменить на линеаризованное уравнение

$$
\frac{d}{d \xi}\left(\xi w_{2}^{\prime}(\xi)\right)=B_{2} \xi\left(\tilde{h}-w_{2}(\xi)\right)-\tilde{\lambda}_{2} \xi
$$

Общее решение этого уравнения имеет вид

$$
w_{2}(\xi)=C_{3} J_{0}\left(\sqrt{B_{2}} \xi\right)+C_{4} Y_{0}\left(\sqrt{B_{2}} \xi\right)+\tilde{h}-\frac{\tilde{\lambda}_{2}}{B_{2}},
$$

где $C_{3}$ и $C_{4}$ - произвольные постоянные. С учетом второго из условий (45) получим $C_{4}=0$ и, следовательно,

$$
\begin{gathered}
w_{2}(\xi)=C_{3} J_{0}\left(\sqrt{B_{2}} \xi\right)+\tilde{h}-\frac{\tilde{\lambda}_{2}}{B_{2}}, \\
w_{2}^{\prime}(\xi)=-C_{3} \sqrt{B_{2}} J_{1}\left(\sqrt{B_{2}} \xi\right) .
\end{gathered}
$$

Отсюда с учетом условия (46) и первого из условий (45) находим

$$
\begin{gathered}
\sqrt{B_{2}} C_{3} J_{1}\left(\sqrt{B_{2}} \xi_{2}\right)=-\gamma_{2}, \\
C_{3} J_{0}\left(\sqrt{B_{2}} \xi_{2}\right)=\frac{\tilde{\lambda}_{2}}{B_{2}} .
\end{gathered}
$$

Подставив в (44) выражение для $w_{2}(\xi)$ и выполнив интегрирование, получим уравнение, связывающее неизвестные $C_{3}, \xi_{2}, \tilde{\lambda}_{2}$,

$$
2 C_{3} \sqrt{B_{2}} \xi_{2} J_{1}\left(\sqrt{B_{2}} \xi_{2}\right)=\tilde{\lambda}_{2}\left(\xi_{2}\right)^{2}-\frac{B_{2}}{\pi} .
$$

В предположении $J_{1}\left(\sqrt{B_{2}} \xi_{2}\right) \neq 0$ из соотношений $(67)$, (68) следует

$$
C_{3}=-\frac{\gamma_{2}}{\sqrt{B_{2}} J_{1}\left(\sqrt{B_{2}} \xi_{2}\right)}, \quad \tilde{\lambda}_{2}=-\sqrt{B_{2}} \gamma_{2} \frac{J_{0}\left(\sqrt{B_{2}} \xi_{2}\right)}{J_{1}\left(\sqrt{B_{2}} \xi_{2}\right)} .
$$


Подставив эти выражения для $C_{3}, \tilde{\lambda}_{2}$ в (69), получим трансцендентное уравнение для нахождения $\xi_{2}$ :

$$
\left\{\frac{J_{0}\left(\sqrt{B_{2}} \xi_{2}\right)}{J_{1}\left(\sqrt{B_{2}} \xi_{2}\right)} \sqrt{B_{2}} \xi_{2}-2\right\} \xi_{2}=-\frac{B_{2}}{\pi \gamma_{2}} .
$$

Решив это уравнение, найдем $\xi_{2}$, далее определим $\tilde{\lambda}_{2}$ и $C_{3}$, а профиль висящей капли найдем из (66). Таким образом, сделан первый шаг процесса линеаризации.

Далее, подставим полученное приближение для функции $w_{2}(\xi)$ в знаменатель левой части уравнения (43). Получим линейное обыкновенное дифференциальное уравнение второго порядка для нахождения следующего приближения и повторим весь процесс, описанный выше. Таков алгоритм процесса линеаризации для висящей капли в предположении сильной смачиваемости.

Пусть $\gamma_{2}=0$ (нулевой угол смачивания). Тогда из (67) следует: либо

1) $C_{3}=0$, из (68) $\tilde{\lambda}_{2}=0$ и (66) дает $w_{2}(\xi) \equiv \tilde{h}$, но условие $(59)$ не выполняется и $w_{2}(\xi) \equiv \tilde{h}$ не является решением поставленной задачи; либо

2) $J_{1}\left(\sqrt{B_{2}} \xi_{2}\right)=0, C_{3} \neq 0$ (в отличие от случая лежащей капли появляется альтернатива).

Рассмотрим вариант 2). В этом случае получим $\xi_{2}=\xi_{2 k}=\beta_{k} / \sqrt{B_{2}}, k=1,2, \ldots$, где $\beta_{k}-k$-ый положительный корень уравнения $J_{1}(z)=0$. Из (69) следует, что $\tilde{\lambda}_{2}=\tilde{\lambda}_{2 k}=\left(B_{2}\right)^{2} / \pi\left(\beta_{k}\right)^{2}$, а из (68) $C_{3}=C_{3 k}=B_{2} /\left(\pi\left(\beta_{k}\right)^{2} J_{0}\left(\beta_{k}\right)\right), k=1,2, \ldots$

Таким образом, в случае висящей капли для нулевого угла смачивания на первом шаге процесса линеаризации в отличие от случая лежащей капли появляются решения следующего вида:

$$
w_{2 k}(\xi)=\frac{B_{2}}{\pi\left(\beta_{k}\right)^{2}}\left(\frac{J_{0}\left(\sqrt{B_{2}} \xi\right)}{J_{0}\left(\beta_{k}\right)}-1\right)+\tilde{h}, k=1,2, \ldots
$$

Проведенные расчеты показывают, что при выборе первого положительного нуля $\left(\beta_{1}=3.8317, J_{1}\left(\beta_{1}\right)=0\right)$ полученное таким образом решение имеет физический смысл.

Построим асимптотику решения задачи первого шага процесса линеаризации при $\gamma_{2} \rightarrow 0$ для висящей капли. Предположим, что величина $\xi_{2}$ имеет такую же асимптотику при $\gamma_{2} \rightarrow+0$, как и соответствующая величина в случае лежащей капли, т. е. $\xi_{2} \sim D_{2} / \sqrt{\gamma_{2}}$, где постоянная $D_{2}=\left(B_{2}\right)^{1 / 4} / \sqrt{\pi}$.

Подставим выбранное асимптотическое представление для $\xi_{2}$ в уравнение (71), умноженное на $\gamma_{2}$. С учетом поведения функций Бесселя при больших значениях аргумента получим

$$
\operatorname{ctg}\left(\sqrt{B_{2}} \xi_{2}-\frac{\pi}{4}\right)\left(D_{2}\right)^{2} \sqrt{B_{2}} \sim-\frac{B_{2}}{\pi}+2 D_{2} \sqrt{\gamma_{2}} .
$$

Следовательно, существует числовая последовательность $\xi_{2 k} \rightarrow+\infty$ при $k \rightarrow+\infty$, на которой

$$
\operatorname{ctg}\left(\sqrt{B_{2}} \xi_{2 k}-\frac{\pi}{4}\right)=-\frac{\sqrt{B_{2}}}{\pi\left(D_{2}\right)^{2}}=-1
$$

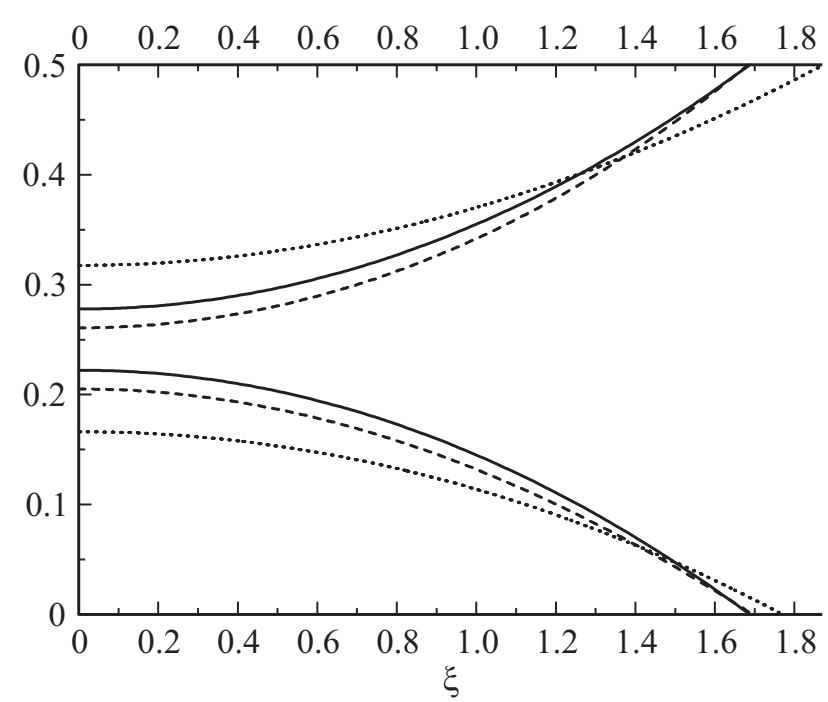

Рис. 3. Результаты расчета профилей лежащей и висящей $(\tilde{h}=0.5)$ капель (число Бонда $B=0.088$, угол смачивания $\theta=15^{\circ}$ ). Сплошная линия - нулевое приближение асимптотического разложения для малого числа Бонда, штриховая сумма нулевого и первого приближений этого разложения, пунктирная - нулевое приближение процесса линеаризации для малого угла смачивания.

\section{Отсюда}

$$
\xi_{2 k}=\frac{(k+1) \pi}{\sqrt{B_{2}}}, \gamma_{2 k}=\frac{\left(B_{2}\right)^{3 / 2}}{(k+1)^{2} \pi^{3}}, k=1,2, \ldots
$$

Далее, найдем $C_{3 k}, \tilde{\lambda}_{2 k}$ по формулам, приведенным выше. А их асимптотика при больших значениях $k$ будет иметь вид

$$
C_{3 k} \sim \frac{(-1)^{k+1} B_{2}}{\pi^{2}(k+1)^{3 / 2}}, \quad \tilde{\lambda}_{2 k} \sim \frac{\left(B_{2}\right)^{2}}{\pi^{3}(k+1)^{2}} .
$$

Следовательно, асимптотика при $\gamma_{2} \rightarrow+0(k \rightarrow+\infty)$ для профиля поверхности висящей капли после первого шага процесса линеаризации будет иметь вид

$$
w_{2 k}(\xi) \sim \frac{(-1)^{k+1} \pi^{1 / 4}\left(\gamma_{2 k}\right)^{3 / 4}}{\left(B_{2}\right)^{1 / 4}} J_{0}\left(\sqrt{B_{2}} \xi\right)+\tilde{h}-\frac{\gamma_{2 k}}{\sqrt{B_{2}}} .
$$

Видно, что максимум модуля асимптотического представления решения в каждой точке стремится к $\tilde{h}$ как $\left(\gamma_{2 k}\right)^{3 / 4}$ при $\gamma_{2} \rightarrow+0(k \rightarrow+\infty)$.

На рис. 3 приведены результаты расчетов профилей лежащей и висящей капель, выполненных с помощью формул, полученных выше.

\section{Заключение}

В работе даны вариационные постановки задач о лежащей и висящей каплях с учетом силы тяжести в осесимметричном случае (в цилиндрической системе координат). Для этих задач построены нулевое и первое 
приближения асимптотического разложения для случая малых чисел Бонда. Кроме того, дан алгоритм линеаризации этих задач при условии сильной смачиваемости и получены приближенные формулы, справедливые для любого значения числа Бонда. Построена асимптотика решений для случая, когда угол смачивания стремится к нулю. Показано, что в случае нулевого угла смачивания для висящей капли существуют решения (в отличие от лежащей капли).

\section{Список литературы}

[1] Финн Р. Равновесные капиллярные поверхности. Математическая теория. М.: Мир, 1989. 312 с.

[2] Антонов П.И., Затуловский Л.М., Костыгов А.С. и др. Получение профилированных монокристаллов и изделий способом Степанова / Под ред. В.Р. Регеля, С.П. Никанорова. Л.: Наука, 1981. 280 с.

[3] Гюнтер Н.М. Курс вариационного исчисления. Л.-М.: ОГИЗ, 1941. $308 \mathrm{c}$.

[4] Галактионова Н.Е., Галактионов Е.В., Тропп Э.А. // Изв. РАН, сер. физ. 2009. Т. 73. Вып. 10. С. 1393-1397.

[5] Френкель Я.И.// ЖЭТФ. 1948. Т. 18. Вып. 7. С. 659-665.

[6] Канчукоев В.3.// Письма в ЖТФ. 2004. Т. 30. Вып. 2. C. $12-16$. 